\title{
COMPARAÇÃO DO USO DE MODELOS DIGITAIS DE ELEVAÇÃO EM MAPEAMENTO DIGITAL DE SOLOS EM DOIS IRMÃOS, RS, BRASIL ${ }^{(1)}$
}

\author{
Rodrigo Teske $^{(2)}$, Elvio Giasson $^{(3)} \&$ Tatiane Bagatini $^{(4)}$
}

\begin{abstract}
RESUMO
Os modelos digitais de elevação (MDEs) são fontes fundamentais para correlacionar a ocorrência e distribuição de solos com a paisagem pelo mapeamento digital de solos (MDS). A influência dos tipos e das resoluções dos MDEs na capacidade de predição dos modelos preditores de classes de solo ainda é pouco estudada. Neste estudo, foram avaliados e comparados os efeitos de diferentes MDEs na predição de ocorrência de unidades de mapeamento de solo (UM). Foram correlacionados 12 atributos do terreno derivados de diferentes MDEs com a ocorrência de UM. Os MDEs utilizados foram os oriundos dos projetos SRTM v4.1, ASTER GDEM v2, TOPODATA e Brasil em Relevo, e os MDEs gerados a partir de curvas de nível na escala de 1:50.000, com resoluções de 30 e $90 \mathrm{~m}$. Os modelos preditores foram treinados por árvore de decisão (Simple Cart) com dados amostrados em 4.280 pontos aleatórios contendo informações dos solos extraídos de um mapa convencional de solos na escala 1:20.000 e 12 atributos do terreno derivados de seis MDEs com tamanhos de pixel de $30 \mathrm{e} 90 \mathrm{~m}$. A validação dos modelos preditores de UM foi realizada com a totalidade dos dados da área. Os atributos do terreno que melhor explicaram a ocorrência das UM foram elevação, declividade, comprimento de fluxo e orientação das vertentes. Os MDEs com tamanho de pixel de $30 \mathrm{~m}$ geraram correlações solo-paisagem menos acuradas. Os modelos preditores mais acurados e com maior número de UM estimadas foram os gerados a partir dos MDEs com resolução espacial de 90 m (SRTM v4.1 e CN90), sendo esses os MDEs mais indicados para o MDS, quando predominarem relevos plano e suave ondulado.

Termos de indexação: atributos do terreno, resolução espacial, tamanho de pixel, árvore de decisão.
\end{abstract}

(1) Parte da Tese de Doutorado do primeiro autor. Estudo financiado pela CAPES e pelo CNPq. Recebido para publicação em 26 de novembro de 2013 e aprovado em 2 de junho de 2014.

(2) Doutorando em Ciência do Solo, Departamento de Solos - DS, Faculdade de Agronomia - FA, Universidade Federal do Rio Grande do Sul - UfRGS. Av. Bento Gonçalves, 7712. CEP 91540-000 Porto Alegre (RS). Bolsista CAPES. E-mail: rodrigoteske.agr@gmail.com

(3) Professor Associado, DS, FA, UFRGS. E-mail: giasson@ufrgs.br

(4) Doutoranda em Ciência do Solo, DS, FA, UFRGS. Bolsista CNPq. E-mail: tatibagatini@yahoo.com.br 


\title{
SUMMARY: COMPARISON OF THE USE OF DIGITAL ELEVATION MODELS IN DIGITAL MAPPING OF SOILS OF DOIS IRMÃOS, RS, BRAZIL
}

\begin{abstract}
Digital elevation models (DEM) are fundamental sources for correlating the occurrence and distribution of soils in the landscape by digital soil mapping (DSM). The influence of the types and resolutions of DEMs on the predictive capability of prediction models of soil classes has not been greatly studied. This study evaluated and compared the effects of different DEMs on the prediction of occurrence of soil mapping units (MU). Twelve predictor variables derived from different DEMs were correlated with the occurrence of MU. The DEMs used were those from the SRTM 4.1, ASTER GDEM V2, TOPODATA, and Brasil em Relevo projects, and the DEMs generated from contour lines on the scale of 1:50,000, with resolutions of 30 and $90 \mathrm{~m}$. The predictive models were guided by a decision tree (Simple Cart) with data sampled at 4,280 random points containing information extracted from soils of a conventional soil map at a scale of 1:20,000 and 12 terrain attributes derived from six DEMs with pixel sizes of 30 and $90 \mathrm{~m}$. The validation of MU prediction models was performed with all the data from the area. The terrain attributes that best explained the occurrence of MU were elevation, slope, flow length, and aspect. The DEMs with $30 \mathrm{~m}$ pixel size generated less accurate soil-landscape correlations. The most accurate prediction models with a higher number of estimated MU were generated from the DEMs with spatial resolution of $90 \mathrm{~m}$ (SRTM 4.1 and CN90). These are the DEMs most suitable for DSM when flat and slightly rolling terrain predominate.
\end{abstract}

Index terms: terrain properties, spatial resolution, pixel size, decision tree.

\section{INTRODUÇÃO}

Um modelo digital de elevação (MDE) é definido como a representação quantitativa digital da variação contínua do relevo sobre o espaço (Moore et al., 1993). Como a relação entre a ocorrência dos solos e os atributos da paisagem é um conceito consolidado na ciência do solo, os MDEs são fundamentais para o mapeamento digital de solos (MDS), pois são fontes digitais disponíveis adequadas para correlacionar a ocorrência e distribuição de solos com as feições do terreno (Florinsky, 2012). Em razão da fundamental influência que o relevo exerce na formação dos solos e da ampla disponibilidade dos MDEs (Behrens et al., 2010), os atributos do terreno derivados dos MDEs são comumente utilizados como variáveis preditoras ao estabelecer as relações solo-paisagem pelas técnicas do MDS (Lagacherie \& McBratney, 2007). Dessa forma, o desenvolvimento do MDS está diretamente relacionado com os avanços tecnológicos na captação, geração e disponibilidade dos MDEs (Cavazzi et al., 2013).

A partir de um MDE, podem ser derivados os atributos do terreno primários e secundários. Os primários são gerados diretamente a partir do MDE como elevação, declividade e orientação das vertentes, entre outros. Os secundários são resultantes de análises geomorfométricas mais elaboradas da paisagem como é o caso do índice de umidade topográfica, índice de convergência topográfica e índice de posição topográfica (Wilson \& Gallant, 2000; Tagil \& Jenness, 2008). Contudo, os MDEs adquiridos por sensores remotos orbitais apresentam erros e têm sido constantemente melhorados e, ou, refinados com uso de algoritmos que filtram os picos anômalos, eliminação de falsas depressões e pontos ausentes de informação e melhorias da definição de corpos d'água e linhas de encosta (Rodrigues et al., 2010; Wilson, 2012). Os projetos Brasil em Relevo (Miranda, 2005) e o TOPODATA (Valeriano \& Rossetti, 2012) visam aprimorar os dados originais do SRTM para o Brasil, corrigindo as falhas e distorções ou refinando o tamanho do pixel. Em nível mundial, é disponibilizado o MDE SRTM v4.1 (Shuttle Radar Topographic Mission v4.1) com preenchimento dos pontos vazios originais do SRTM (Jarvis et al., 2008) e a segunda versão do projeto Advanced Spaceborne Thermal Emission and Reflection Radiometer (ASTER GDEM v2) (Meyer et al., 2012).

Segundo Cavazzi et al. (2013), o meio ambiente não pode ser estudado, modelado ou visualizado em toda a sua complexidade e seus detalhes e, por isso, utiliza-se do efeito de escala para selecionar e generalizar as informações. Segundo McBratney et al. (2003), em se tratando de mapas digitais de solos, diferentemente da cartografia convencional, a escala é um conceito complexo, sendo melhor substituída por resolução espacial e tamanho de pixel. Por causa da disponibilidade de diferentes tipos de MDEs, pode-se acreditar que os MDEs com maior resolução espacial, menor tamanho de pixel, possibilitam melhor representação das características da paisagem e, com isso, geram modelos preditores de ocorrência de tipos de solos mais acurados. McBratney et al. (2003) apresentaram aproximações compatíveis entre o espaçamento de pixel e as escalas de mapas. Todavia, além dessas relações cartográficas, Smith et al. (2006) relataram que a acurácia dos trabalhos com MDS pode ser influenciada pelas características da paisagem e pela forma como essas informações são representadas pelos MDEs.

Os estudos que abordam a influência dos tipos e as resoluções dos MDEs sobre modelos preditivos de solos 
são mais comumente encontrados quando envolvem a predição espacial de propriedades dos solos do que os de predição de ocorrência de solo (Behrens et al., 2010). Em trabalho recente, Giasson et al. (2013) relataram que a comparação entre três MDEs, com pixel de 30 e $90 \mathrm{~m}$, utilizados para a predição de unidades de mapeamento de solos (UM), não foi conclusiva, pois a diferença na capacidade de predição dos modelos foi muito pequena. Ao utilizar análise wavelet e variáveis preditoras derivadas do MDE TOPODATA, ten Caten et al. (2012) sugeriram que a resolução espacial a ser utilizada no MDS deve ser entre $32 \mathrm{e} 40 \mathrm{~m}$. Em estudo realizado por Cavazzi et al. (2013), os autores demonstraram que, em áreas com relevo menos declivoso, os MDEs com menores resoluções espaciais $(140 \mathrm{~m})$ possibilitaram gerar modelos de predição de solos mais acurados.

Nesse contexto, foram avaliados e comparados modelos de predição de ocorrência de solos gerados por árvore de decisão, correlacionando as UM extraídas de um mapa convencional de solos na escala de 1:20.000 e 12 atributos do terreno derivados de seis tipos e resoluções de MDEs.

\section{MATERIAL E MÉTODOS}

A área de estudo foi o município de Dois Irmãos, situado na região fisiográfica da Encosta Inferior do nordeste do Estado do Rio Grande do Sul. Essa região é caracterizada por compor parte da borda do relevo do Planalto das Araucárias, cuja geologia é representada pelas rochas basálticas da Formação Serra Geral e pelos arenitos da Formação Botucatu (GeoBank, 2014). O município ocupa uma área aproximada de $68,5 \mathrm{~km}^{2}$ e apresenta quatro classes principais de relevo: plano, suave ondulado, ondulado e forte ondulado; entretanto, predominam as formas de relevo menos íngremes (plano e suave ondulado). O município situa-se no limite de dois tipos climáticos, segundo a classificação do clima de Köppen: Cfa (clima subtropical úmido quente) e Cfb (clima subtropical úmido temperado). Embora demais fatores estejam relacionados com a formação dos solos, o relevo foi considerado como o principal fator diferenciador entre os tipos de solo, por condicionar os fluxos de água, a drenagem, o acúmulo de materiais e os processos erosivos. Por isso, foram utilizados apenas atributos do relevo para avaliar e comparar a influência dos diferentes MDEs sobre modelos preditores de ocorrência de solos gerados por árvore de decisão.

Os MDEs utilizados para derivar os atributos do terreno estão representados na figura 1 e são: ASTER GDEM v2, com resolução espacial de $30 \mathrm{~m}$, apresenta um acréscimo de 260.000 pares estereoscópicos de imagens em relação à primeira versão do ASTER GDEM (Meyer et al., 2012); SRTM v4.1, disponibilizado para a América do Sul com tamanho de pixel de aproximadamente de $90 \mathrm{~m}$ (Jarvis et al.,
2008); TOPODATA, em que os dados originais do SRTM foram interpolados para refinamento no tamanho do pixel de 90 para $30 \mathrm{~m}$, além de inclusão de informação nos pontos vazios (Valeriano \& Rossetti, 2012); Brasil em Relevo (BR_Relevo), com o mesmo tamanho de pixel que o dado original do SRTM (90 m), mas com correções de cortes e preenchimentos (Miranda, 2005) e MDEs gerados nas resoluções de $30 \mathrm{~m}$ (CN30) e $90 \mathrm{~m}$ (CN90), com auxílio da função topo to raster do programa ArcGis 9.3 (ESRI, 2009) a partir das curvas de nível com equidistância vertical de $20 \mathrm{~m}$ disponibilizadas em Hasenack \& Weber (2010). Para comparar e avaliar qualitativamente os MDEs, foram gerados dois perfis de elevação nos sentidos norte-sul (A-A') e leste-oeste (B-B') (Figura 1), a partir de dois transectos traçados em ambiente de SIG.

A correlação entre as características da paisagem e a distribuição das UM no município de Dois Irmãos foi realizada pelo treinamento do algoritmo de árvore de decisão Simple Cart, a partir de informações coletadas em ambiente de sistema de informação geográfica (SIG) da variável resposta (UM) e das variáveis preditoras (atributos do terreno). As UM foram extraídas do mapa de solos na escala 1:20.000 (Figura 2), que faz parte do levantamento de reconhecimento com detalhes dos solos do município de Dois Irmãos (Klamt et al., 1993).

A partir de cada MDE, foram gerados 12 atributos do terreno, sendo nove primários (elevação, declividade, curvatura, curvatura em perfil, curvatura planar, direção do fluxo, comprimento do fluxo, acúmulo do fluxo e orientação das vertentes) e três secundários (índice de umidade topográfica, índice de convergência topográfica e índice de posição topográfica). A variável distância dos rios foi calculada a partir da rede hidrográfica da base cartográfica vetorial 1:50.000 (Hasenack \& Weber, 2010). Para gerar os modelos de árvores de decisão (AD), foram coletados os dados dos atributos do terreno e das UM em 4.280 pontos amostrais distribuídos aleatoriamente por toda a área de estudo. Essa amostragem corresponde a um ponto para cada área mínima mapeável para a escala 1:20.000 (1 ponto/1,6 ha). Os dados foram tabelados e exportados para o programa de mineração de dados Weka 3.6.3 (Hall et al., 2009), a fim de serem treinados pelo algoritmo Simple Cart com número de elementos no nó final (M) igual a 2 (M2) e 11 (M11). Foram testados diversos valores para M (dados não apresentados); $M$ igual a 11 (M11) possibilitou gerar $\mathrm{AD}$ menores e menos complexas sem reduzir demasiadamente a acurácia dos modelos e a quantidade das UM preditas.

A validação dos modelos preditores foi realizada com a totalidade dos dados da área de estudo. Para isso, foram coletadas todas as informações das variáveis preditoras (atributos do terreno) e das variáveis reposta (UM) ocorrentes em todos os pixels. Esses dados foram tabulados e importados para o 


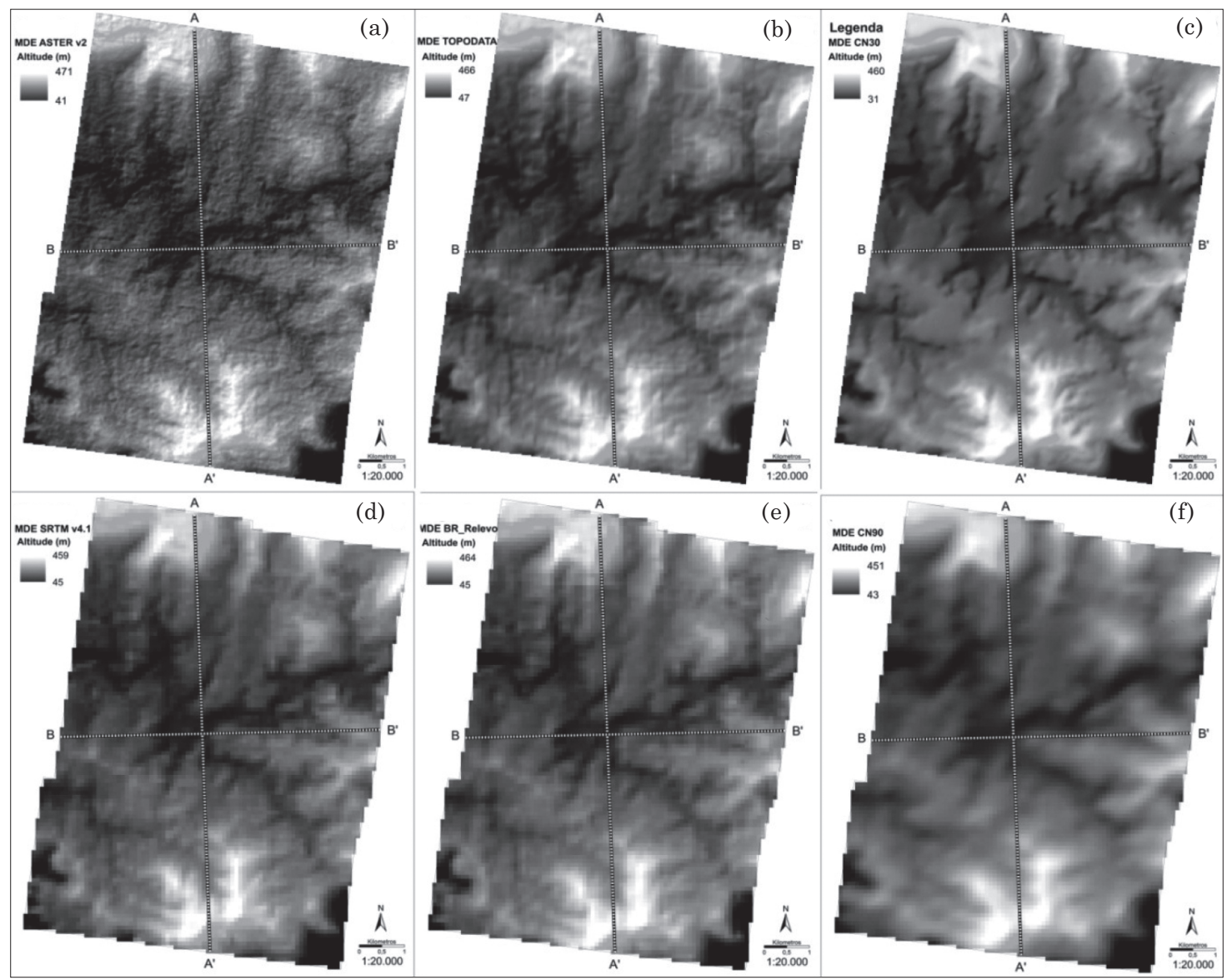

Figura 1. Transectos e modelos digitais de elevação utilizados: (a) ASTER GDEM v2; (b) TOPODATA; (c) MDE CN30; (d) SRTM v4.1; (e) BR_Relevo; e (f) CN90.

programa Weka 3.6.3 (Hall et al., 2009) para aplicação do método de validação de modelos com dados independentes (Supplied test set). O método de validação com dados independentes é realizado por comparações em matriz de erros (Congalton, 1991), confrontando a predição de ocorrência de solos com a distribuição original (Rossiter, 2004). Assim, foram obtidas as estimativas da acurácia geral (AG), que é a proporção de instâncias corretamente classificadas, e o índice Kappa (Cohen, 1960), que mede as concordâncias compensando o acaso.

\section{RESULTADOS E DISCUSSÃO}

A representação do relevo da área de estudo foi diferenciada para cada MDE utilizado, que apresentaram diferentes valores mínimos e máximos para elevação (Figura 2). Os maiores valores de elevação foram encontrados nos MDEs ASTER GDEM v2 e TOPODATA; e os menores, nos MDEs CN30 e ASTER GDEM v2. As maiores amplitudes nos valores da elevação foram encontradas nos MDEs com resolução espacial de $30 \mathrm{~m}$, principalmente no $\mathrm{MDE}$
ASTER GDEM v2. Segundo Guth (2010), entre os MDEs gerados a partir de dados obtidos por sensores remotos orbitais, o ASTER GDEM apresenta maior quantidade de artefatos e variações superficiais, que são consideradas e quantificadas como valores de elevação (Rodrigues et al, 2010; Wilson, 2012).

Os perfis de elevação dos transectos (Figura 3) demonstraram que os MDEs gerados a partir da carta topográfica na escala de 1:50.000 (CN30 e CN90) apresentaram os menores valores de elevação em praticamente toda extensão destes transectos. Esses resultados estão em concordância com o trabalho de Chagas et al. (2010), os quais, ao compararem diferentes MDEs com resolução espacial de $30 \mathrm{~m}$, constataram que o MDE gerado a partir de curvas de nível, com equidistância vertical de $20 \mathrm{~m}$ na escala de 1:50:000, permitiu representar melhor as características do terreno e apresentou menores valores de elevação do que os MDEs ASTER GDEM e SRTM. Os MDEs originados de dados orbitais do SRTM v4.1 e ASTER GDEM v2 apresentaram os maiores valores de elevação e as maiores oscilações nesses valores, indicando, assim, a influência das variações superficiais causadas por edificações e árvores. Embora alguns trabalhos indiquem que o MDE ASTER 
GDEM apresenta algumas vantagens em relação aos produtos do SRTM, como a resolução espacial de $30 \mathrm{~m}$ (Rodrigues et al., 2010), as maiores oscilações nos valores de elevação do MDE ASTER GDEM v2 indicam que esse MDE evidencia maior quantidade de artefatos do que o SRTM, sendo agravados em regiões de relevo mais suave, resultando numa pior representação do relevo, como constatado por Guth (2010).

Os resultados da avaliação da acurácia dos modelos indicaram que as predições de ocorrência de UM foram influenciadas tanto pelo tipo como pela resolução espacial dos MDEs (Quadro 1). Os modelos preditores gerados a partir dos MDEs com pixel de $90 \mathrm{~m}$, utilizando M2, foram os mais acurados. Esses modelos apresentaram as maiores $\mathrm{AD}$, o que permitiu estabelecer melhores correlações entre as características do terreno e as UM, resultando na predição de todas as 15 UM presentes no mapa convencional de solos. A correlação das UM com os atributos do terreno derivados do MDE CN90 resultou em um modelo preditivo com AG de 54,71\% e índice Kappa de 0,46. O modelo preditor de UM gerado com os dados do MDE SRTM v4.1 apresentou uma AG de 52,69 \% e índice Kappa igual a 0,43. Esses resultados

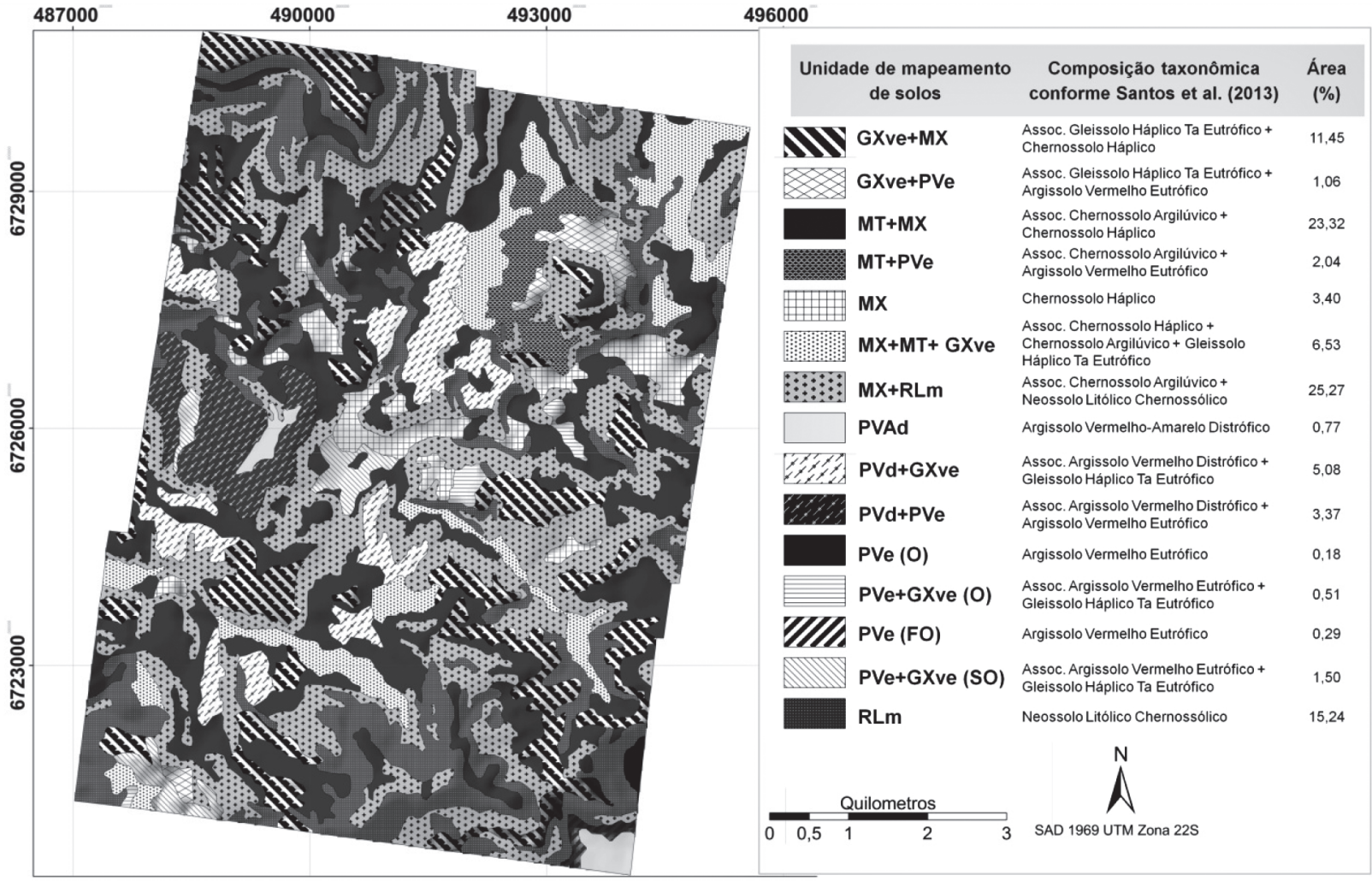

Figura 2. Mapa de solos do município de Dois Irmãos, Rio Grande do Sul. Fonte: Klamt et al. (1993).

Quadro 1. Resultados da avaliação da acurácia dos modelos de predição de ocorrência de unidades de mapeamento de solos usando seis modelos digitais de elevação (MDE)

\begin{tabular}{|c|c|c|c|c|c|c|c|c|c|}
\hline \multirow{2}{*}{ MDE } & \multirow{2}{*}{ Resolução espacial } & \multicolumn{4}{|c|}{$\mathbf{M}^{(1)} 2$} & \multicolumn{4}{|c|}{ M11 } \\
\hline & & $A G^{(2)}$ & Kappa & Tam..$^{(3)}$ & $\mathbf{N}^{\circ} \mathbf{U} \mathbf{M}^{(4)}$ & $A G^{(2)}$ & Kappa & Tam. ${ }^{(3)}$ & $\mathbf{N}^{\mathbf{o}} \mathbf{U} \mathbf{M}^{(4)}$ \\
\hline & $\mathrm{m}$ & $\%$ & & & & $\%$ & & & \\
\hline ASTER GDEM v2 & 30 & 37,16 & 0,23 & 141 & 11 & 36,66 & 0,22 & 73 & 9 \\
\hline TOPODATA & 30 & 45,98 & 0,35 & 459 & 13 & 44,28 & 0,32 & 117 & 9 \\
\hline CN30 & 30 & 45,65 & 0,34 & 431 & 14 & 43,61 & 0,31 & 169 & 12 \\
\hline BR_Relevo & 90 & 47,93 & 0,37 & 921 & 15 & 44,48 & 0,33 & 195 & 12 \\
\hline SRTM v4.1 & 90 & 52,69 & 0,43 & 999 & 15 & 47,06 & 0,36 & 107 & 11 \\
\hline CN90 & 90 & 54,71 & 0,46 & 985 & 15 & 46,61 & 0,35 & 147 & 13 \\
\hline
\end{tabular}

(1) M: número de elementos no nó final da árvore de decisão; ${ }^{(2)} \mathrm{AG}$ : acurácia geral; ${ }^{(3)}$ Tam.: tamanho da árvore de decisão; e ${ }^{(4)} \mathrm{N}^{\mathrm{o}} \mathrm{UM}$ : quantidade de unidades de mapeamento de solos estimada em cada modelo preditor. 


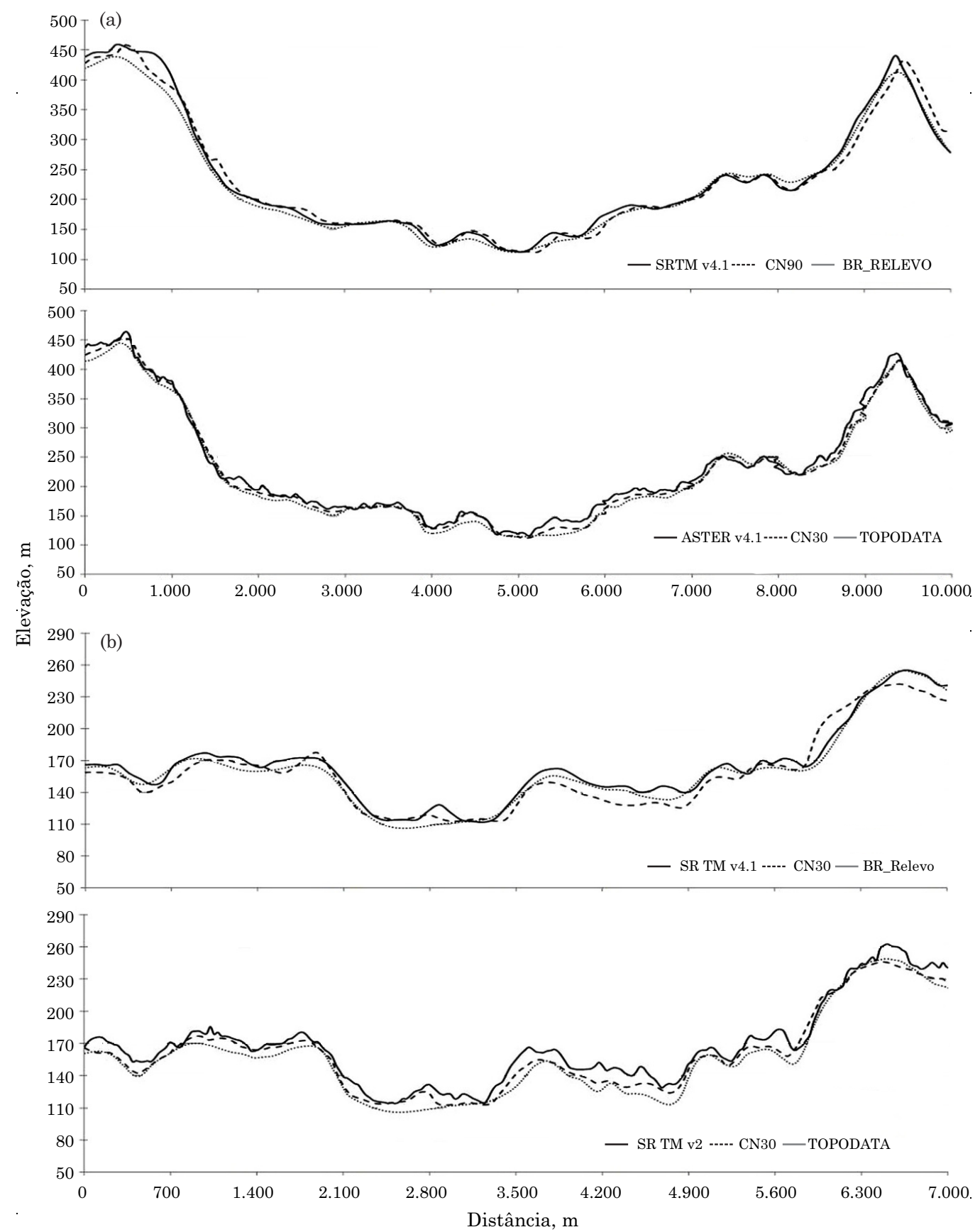

Figura 3. Perfis de elevação no transecto A-A`(a) e no transecto B-B`(b) dos modelos digitais de elevação com resoluções espaciais de 90 m (SRTM v4.1, CN90 e BR_Relevo) e 30 m (ASTER v2, CN30 e TOPODATA).

da acurácia são comparáveis e consistentes com demais estudos com MDS, como os resultados de Cavazzi et al. (2013), em que os mapas preditores de classes de solo apresentaram acurácia entre 35 e $60 \%$ ao utilizar MDEs gerados a partir de curvas de nível, na escala de 1:50:000. Behrens et al. (2010) analisaram digitalmente um terreno em multiescala, utilizando filtros com dimensões que variaram de $3 \times$ 3 até $31 \times 31$ pixels e encontraram uma acurácia geral de $54 \%$ na área de validação. Há, também, os resultados de Giasson et al. (2013), os quais, ao compararem diferentes algoritmos de treinamento e diferentes MDEs, encontraram valores de acurácia entre 50,0 e $57,3 \%$.

Nos modelos preditores gerados a partir dos MDEs com tamanho de pixel de $30 \mathrm{~m}$ e M2, a quantidade de UM preditas variou conforme o tipo de captação dos dados de elevação. Ao utilizar os atributos do terreno derivados do MDE CN30, o modelo preditor estimou a ocorrência de 14 UM; com o MDE TOPODATA foram preditas $13 \mathrm{UM}$ e com uso dos atributos derivados do MDE ASTER GDEM v2 foram estimadas 11 UM. O 
tamanho das $\mathrm{AD}$ geradas com os MDEs de $30 \mathrm{~m}$ foi consideravelmente menor do que as $\mathrm{AD}$ geradas com os MDEs de $90 \mathrm{~m}$ e M2, principalmente para o MDE ASTER GDEM v2, que apresentou as maiores oscilações nos valores da elevação.

Os modelos preditores de UM gerados com M11 apresentaram $\mathrm{AD}$ menores, foram menos acurados e estimaram menos UM do que os gerados com M2. Essas diferenças foram mais expressivas nos modelos mais acurados (CN90 e SRTM v4.1), indicando que a redução no tamanho das $\mathrm{AD}$ pelo aumento do valor de $M$ pode prejudicar a capacidade preditiva de modelos, principalmente os mais acurados. Todavia, os modelos gerados a partir dos MDEs com pixel de 90 m (CN90 e SRTM v4.1) permaneceram sendo os mais acurados, enquanto o modelo preditor de UM, gerado a partir do MDE ASTER GDEM v2, ficou sendo o menos acurado e com a menor quantidade de UM preditas. Assim, indiferentemente do valor de $\mathrm{M}$ adotado, os modelos de predição de ocorrência de UM gerados a partir dos MDEs com tamanho de pixel de 90 m estabeleceram as melhores correlações solo-paisagem e, com isso, geraram os modelos de predição de ocorrência de UM mais acurados.

Os atributos do terreno derivados dos MDEs (elevação, declividade, comprimento de fluxo e orientação das vertentes) foram os que melhor explicaram a relação solo-paisagem em todos os modelos preditores. A importância dessas variáveis é constatada por diversos autores (Behrens et al., 2010; ten Caten et al., 2012; Giasson et al., 2013), os quais comumente têm identificado a elevação e a declividade entre os principais atributos do terreno usados no MDS. Utilizando os MDEs ASTER GDEM, TOPODATA e SRTM, ten Caten et al. (2012) e Giasson et al. (2013) constataram que, além da elevação e da declividade, a curvatura, a curvatura em perfil, a direção de fluxo, o acúmulo de fluxo e o índice de umidade topográfica também apresentaram contribuição expressiva para explicar a ocorrência de classes de solo. Isso está relacionado ao fato de a elevação apresentar importante papel na definição do clima local e dos atributos declividade, orientação das vertentes e comprimento de fluxo, por influenciar a velocidade dos fluxos superficial e subsuperficial de água, a evapotranspiração, insolação, o teor de água no solo, os processos erosivos e deposicionais, influenciando, assim, os processos pedogenéticos, as propriedades e o potencial agrícola dos solos (Moore et al., 1993; Wilson \& Gallant, 2000).

Ao analisar os dados utilizados para treinamento em gráficos de caixa - box plot - (Figura 4), pode-se observar que as amostragens evidenciaram dados da elevação muito semelhantes entre os MDEs de $30 \mathrm{~m}$ (representados pelo MDE ASTER GDEM v2) e MDEs de $90 \mathrm{~m}$ (representados pelo MDE CN90). Os dados da elevação, analisados pelas amplitudes média e mediana foram diferenciados entre as UM, demonstrando a alta relação da distribuição das UM com as cotas altimétricas. Todavia, alguns delineamentos como a
UM MT+MX, a UM GXve+MX, a UM RLm e a UM MX+RLm ocorrem em variadas elevações, apresentando maiores amplitudes e mais valores extremos (outliers), sendo um dos motivos para seleção dos demais atributos do terreno, como a declividade e o comprimento de fluxo, na construção das $\mathrm{AD}$ e da geração dos modelos preditores.

A declividade é considerada como um atributo local, sendo calculada com base nos valores da elevação e no tamanho dos seus pixels vizinhos $(3 \times 3$ pixeis $)$ (Florinsky, 2012). Ao utilizar os MDEs com maior tamanho de pixels, os valores da declividade foram menores para os mesmos valores de elevação. Isso pode ser observado nos gráficos de caixas, em que o MDE ASTER GDEM v2 apresentou os maiores valores de declividade (95\%), enquanto para o MDE CN90 o valor máximo para a declividade foi de $23 \%$. Em relação ao comprimento de fluxo, esse é considerado como um atributo não local e é uma medida da distância ao longo do trajeto de fluxo, determinada pela direção do fluxo, a partir de uma dada célula até a sua saída da bacia de drenagem (Florinsky, 2012). Os valores para o comprimento de fluxo, assim como a declividade, foram diferentes entre os MDEs de 30 e $90 \mathrm{~m}$. No MDE ASTER GDEM, os valores desse atributo foram menores, pois percorreram menores distâncias, menor tamanho de pixel, e se apresentaram mais assimétricos e com maior quantidade de valores extremos dentro de cada UM do que no MDE CN90, que, por sua vez, evidenciou maiores diferenças nos valores de média e mediana entre as UM. Dessa forma, os MDEs com resolução espacial de $90 \mathrm{~m}$ possibilitaram gerar modelos mais acurados, pois demonstraram menores quantidades de valores assimétricos e extremos em cada UM, bem como maior diferenciação dos valores dos atributos (médios e da mediana) entre as UM, permitindo, assim, o modelo preditor realizar separações mais homogêneas dos dados (Rokach \& Maimon, 2008) para cada UM, gerando correlações solo-paisagem mais acuradas.

Esses resultados estão em conformidade com os estudos de Thompson et al. (2001), Smith et al. (2006) e Wu et al. (2008), em que à medida que a resolução espacial do MDE é reduzida (aumenta o tamanho do pixel), a declividade é diminuída, o comprimento do percurso de escoamento é aumentado e os detalhamentos nos atributos do terreno são perdidos. Contudo, os MDEs com menor tamanho de pixel não são necessariamente considerados as melhores fontes de informações para gerar variáveis preditoras de solos (Cavazzi et al. (2013). Isso decorre por apresentarem maior quantidade de dados espacializados e variação acentuada nos valores dos atributos do terreno dentro de cada UM. Em se tratando de uma área com predomínio de relevo mais suave, isso resultou em amplitudes dos dados mais semelhantes entre as UM do que as encontradas com os atributos gerados a partir dos MDEs, com pixel de $90 \mathrm{~m}$. Assim, maior amplitude dos dados dentro de cada UM, juntamente com menor variação nas características da paisagem entre as 

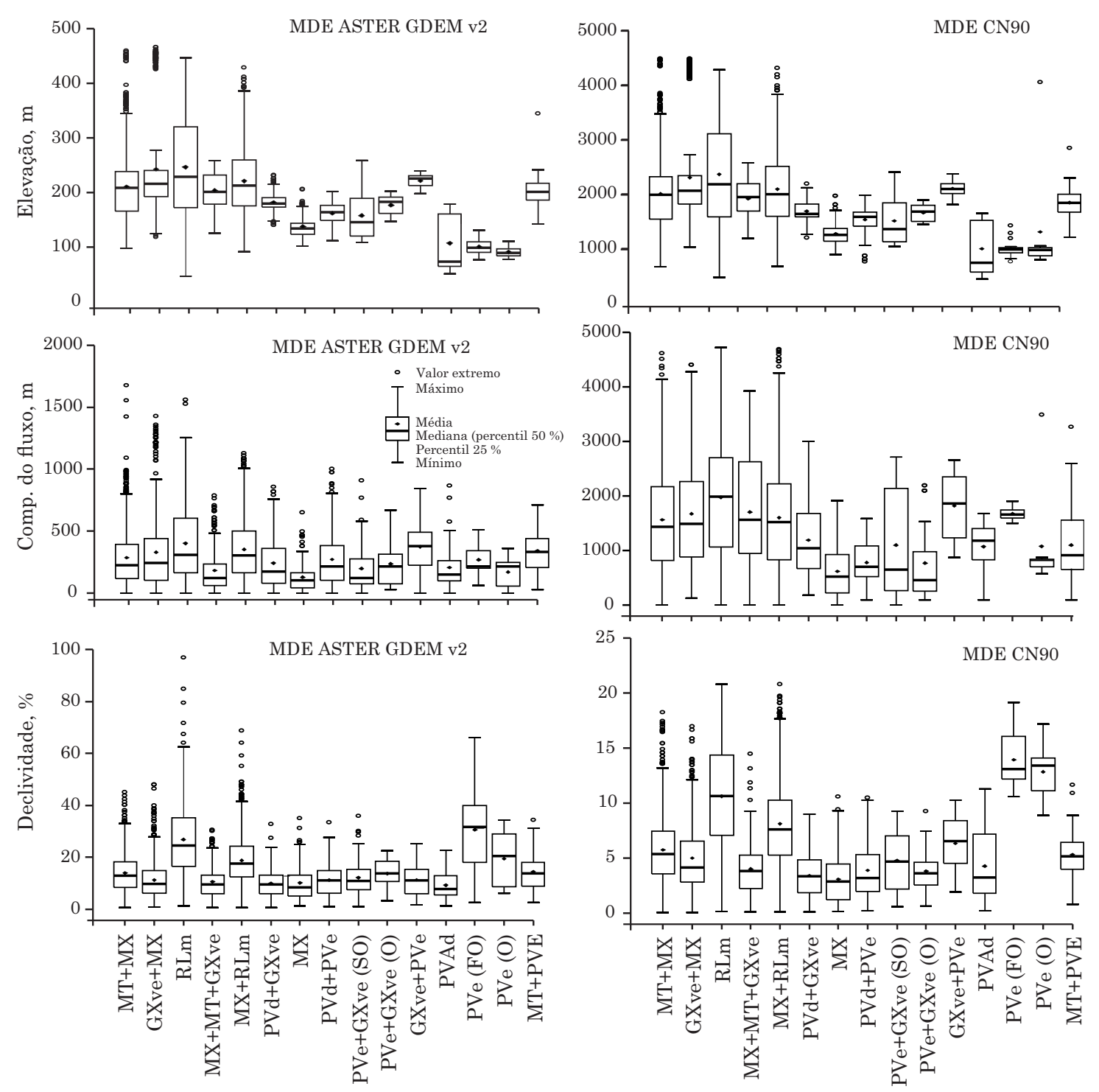

Figura 4. Gráficos de caixas dos dados de elevação, comprimento de fluxo e declividade derivados dos modelos digitais de elevação ASTER GDEM v2 $(30 \mathrm{~m})$ e CN90 $(90 \mathrm{~m})$ para cada unidade de mapeamento constante no mapa convencional de solos.

áreas ocupadas pelas diferentes UM, resultaram modelos preditores menos acurados, pois dificultaram o treinamento do algoritmo e a separação em dados mais homogêneos para cada classe, nesse caso, as UM. Adicionalmente, os MDEs com tamanho de pixel de $30 \mathrm{~m}$ apresentaram mais oscilações na elevação e, por isso, ao predizer a ocorrência de UM para toda a área, os mapas auxiliares das características do relevo apresentaram maior quantidade de oscilações e informações divergentes das regras de classificação geradas, diminuindo ainda mais a sua acurácia.

Além da influência das características da paisagem e da forma como essas são representadas pelos MDEs, as UM representam áreas ambientalmente semelhantes em determinada escala. Assim, ao predizer a ocorrência de UM pelas técnicas do MDS, as UM são também distribuídas para áreas cujas características da paisagem sejam semelhantes conforme os dados de entrada e as correlações realizadas pelo algoritmo. Neste estudo, as informações pedológicas de referência foram extraídas de um mapa convencional de solos na escala de 1:20.000, onde a área mínima mapeável $\left(0,4 \mathrm{~cm}^{2}\right)$ é de $16.000 \mathrm{~m}^{2}(1,6$ ha). Segundo McBratney et al. (2003), o tamanho do pixel sugerido e compatível com mapas na escala de 1:20.000 é de $20 \mathrm{~m}$, variando até $200 \mathrm{~m}$ conforme a área e o objetivo de estudo. Esses autores ainda relataram que uma resolução espacial mínima deve ser de $2 \times 2$ pixels. Assim, os MDEs de $90 \mathrm{~m}$ representaram uma resolução mínima de $32.400 \mathrm{~m}^{2}$ $(180 \times 180 \mathrm{~m})$, enquanto os MDEs de $30 \mathrm{~m}(60 \times 60$ $\mathrm{m}$ ) de somente $3.600 \mathrm{~m}^{2}$, valor esse muito aquém da área mínima mapeável para a escala de 1:20.000. Ao considerar que na área de estudo predominam fases 
de relevo mais suaves e que todas as UM estão representando áreas maiores que a área mínima mapeável, a representação contínua do relevo por MDEs com resolução espacial de 30 m não se mostrou a mais indicada, ao ponto de esses MDEs apresentarem mais oscilações nos valores da elevação (ASTER GDEM) e aumentarem demasiadamente a quantidade de informações espacializadas para uma área com relevo menos complexo, o que dificultou o treinamento do algoritmo e a geração de modelos preditores mais acurados. Dessa forma, a maior diferenciação nos valores dos atributos do terreno dentro das áreas de cada UM tornou-se mais importante do que o maior detalhamento espacializado com uso de MDEs com pixel de $30 \mathrm{~m}$, que não possibilitaram gerar modelos de predição de ocorrência de solos mais acurados.

De forma geral, os resultados da acurácia dos modelos preditores foram satisfatórios e comparáveis com demais estudos de predição categórica de solos (classes ou unidades de mapeamento de solos). Assim, as correlações solo-paisagem geradas por $\mathrm{AD}$ (algoritmo Simple Cart) para predizer a ocorrência de UM para o município de Dois Irmãos, em que predominam fases de relevo plana e suave ondulada, foram melhores quando utilizados atributos do terreno derivados dos MDEs com resolução espacial de $90 \mathrm{~m}$, principalmente com o MDE do projeto SRTM v4.1 e MDE gerado a partir de curvas de nível (CN90).

\section{CONCLUSÕES}

1. Os atributos derivados dos MDEs, que melhor explicaram a distribuição espacial das UM, para uma área com predomínio de fases do relevo menos complexo, foram a elevação, a declividade, o comprimento de fluxo e a orientação das vertentes, com o uso de árvores de decisão.

2. Os MDEs com maior resolução espacial, oriundos de sensores remotos orbitais, apresentaram maiores oscilações nos valores da elevação e geraram correlações solo-paisagem menos acuradas e menor quantidade de unidades de mapeamento preditas.

3. Os MDEs com resolução espacial de $90 \mathrm{~m}$ (SRTM v4.1 e CN90) permitiram gerar modelos preditores de ocorrência de UM mais acurados e com maior número de UM preditas, em áreas que predominam relevo plano a suave ondulado.

\section{AGRADECIMENTOS}

À Coordenação de Aperfeiçoamento de Pessoal de Nível Superior (CAPES), pela bolsa de doutorado para o primeiro autor; e ao Conselho Nacional de Desenvolvimento Científico e Tecnológico (CNPq), pela bolsa de produtividade em pesquisa concedida ao segundo autor e pela bolsa de doutorado para a terceira autora.

\section{LITERATURA CITADA}

BEHRENS, T.; ZHU, A.X.; SCHMIDT, K. \& SCHOLTEN, T. Multi-scale digital terrain analysis and feature selection in digital soil mapping. Geoderma, 155:175-185, 2010.

CAVAZZI, S.; CORSTANJE, R.; MAYR, T.; HANNAM, J. \& FEALY, R. Are fine resolution digital elevation models always the best choice in digital soil mapping? Geoderma, 195/196:111-121, 2013.

CHAGAS, C.S.; FERNANDES FILHO, E.I.; ROCHA, M.F.; CARVALHO JÚNIOR, W. \& SOUZA NETO, N.C. Avaliação de modelos digitais de elevação para aplicação em um mapeamento digital de solos. R. Bras. Eng. Agric. Amb., 14:218-226, 2010.

COHEN, J. A coefficient of agreement for nominal scales. J. Educ. Measur., 20:37-46, 1960

CONGALTON, R.G. A review of assessing the accuracy of classification of remotely sensed data. Remote Sens. Environ., 37:35-46, 1991.

ENVIRONMENTAL SYSTEMS RESEARCH INSTITUTE Inc. - ESRI. ArcGIS. Professional GIS for the desktop, versão 9.3.1 CA. 2009.

FLORINSKY, I. V. Digital terrain analysis in soil science and geology. Amsterdã, Elsevier/Academic Press, 2012. 379p.

GEOBANK - CPRM. Mapa geológico do estado do Rio Grande do Sul. Serviço Geológico do Brasil, 2014. Disponível em: $<$ http://geobank.sa.cprm.gov.br/pls/publico/. Acesso em: 12 abr. 2014.

GIASSON, E.; HARTEMINK, A.E.; TORNQUIST, C.G.; TESKE, R. \& BAGATINI, T. Avaliação de cinco algoritmos de árvores de decisão e três tipos de modelos digitais de elevação para mapeamento digital de solos a nível semidetalhado na Bacia do Lageado Grande, RS, Brasil. Ci. Rural, 43:61-67, 2013.

GUTH, P.L. Geomorphometric comparison of ASTER GDEM and SRTM. A special joint symposium of ISPRS Technical Commission IV \& AutoCarto in conjunction with ASPRS/ CaGIS, 2010.

HALL, M.; FRANK, E.; HOLMES, G.; PFAHRINGER, B.; REUTEMANN, P. \& WITTEN, I.H. The WEKA Data Mining Software: An Update. 2009. (SIGKDD Explorations, 11)

HASENACK, H. \& WEBER, E. Base cartográfica vetorial contínua do Rio Grande do Sul - escala 1:50.000. Porto Alegre, UFRGS, IB - Centro de Ecologia, 2010. DVD-ROM (Série Geoprocessamento, 3)

JARVIS, A.; REUTER, H.I.; NELSON, A. \& GUEVARA, E. Hole-filled SRTM for the globe Version 4, available from the CGIAR-CSI SRTM 90 m Database. 2008. Disponível em: <http://srtm.csi.cgiar.org>. 
KLAMT, E.; SCHNEIDER, P.; COUTO, E.G.; BARCELOS, A.A.; LEIPNITZ, P.R. \& SANTOS, G.I.T. Solos do município de Dois Irmãos, RS: Características, distribuição geográfica e aptidão de uso. Porto AlegreUniversidade Federal do Rio Grande do Sul, 1993. 147p.

LAGACHERIE, P. \& McBRATNEY, A.B. Spatial soil information systems and spatial soil inference systems: perspectives for digital soil mapping. In: LAGACHERIE, P.; McBRATNEY, A.B. \& VOLTZ, M., eds. Digital soil mapping: an introductory perspective. Amsterdam, Elsevier, 2007. p.3-24.

McBRATNEY A.B.; MENDONÇA-SANTOS, M.L. \& MINASNY, B. On digital soil mapping. Geoderma, 117:3-52, 2003.

MEYER, D.J.; TACHIKAWA, T.; ABRAMS, M.; CRIPPEN, R.; KRIEGER, T.; GESCH, D. \& CARABAJAL, C. Summary of the validation of the second version of the ASTER GDEM, Int. Arch. Photogramm. Remote Sens. Spatial Inf. Sci., XXXIX-B4:291-293, 2012.

MIRANDA, E.E. Brasil em Relevo. Campinas, Embrapa Monitoramento por Satélite, 2005.

MOORE, I.D.; GESSLER, P.E.; NIELSEN, G.A. \& PETERSON, G.A. Soil attribute prediction using terrain analysis. Soil Sci. Soc. Am. J., 57: 443-452, 1993.

RODRIGUES, T.L.; DEBIASI, P. \& SOUZA, R.F. Avaliação da adequação dos produtos ASTER GDEM no auxílio ao mapeamento sistemático brasileiro. In: SIMPÓSIO BRASILEIRO DE CIÊNCIAS GEODÉSICAS E TECNOLOGIAS DA GEOINFORMAÇÃO, 3., 2010, Anais..., Recife, PE, Embrapa, 2010. CD-ROM

ROKACH, L. \& MAIMON, O.Z. Data mining with decision trees: theory and applications. Londres, World Scientific, 2008. 244p.
ROSSITER, D. G. Technical Note: Statistical methods for accuracy assessment of classified thematic maps. Enschede (NL): International Institute for Geoinformation Science \& Earth Observation (ITC). 25:92, 2004. 107p.

SMITH, M.P.; ZHU, A.-X.; BURT, J.E. \& STILES, C. The effects of DEM resolution and neighborhood size on digital soil survey. Geoderma, 137:58-69, 2006.

TAGIL, S. \& JENNESS, J. GIS-based automated landform classification and topographic, landcover and geologic attributes of landforms around the Yazoren Polje, Turkey. J. Appl. Sci., 8:910-921, 2008.

ten CATEN, A.; DALMOLIN, R.S.D.; PEDRON, F.A. \& MENDONCA-SANTOS, M.L. Spatial resolution of a digital elevation model defined by the wavelet function. Pesq. Agropec. Bras., 47:449-457, 2012.

THOMPsOn, J.A.; BELL, J.C. \& BUTLER, C.A. Digital elevation model resolution: effects on terrain attribute calculation and quantitative soil-landscape modeling. Geoderma, 100: 67-89, 2001.

VALERIANO, M.M. \& ROSSETTI, D.F. Topodata: Brazilian full coverage refinement of SRTM data. Appl. Geogr., 32:300-309, 2012.

WILSON, J. P. Digital terrain modeling. Geomorphology, 137:107-121, 2012.

WILSON, J.P. \& GALLANT, J.C. Terrain Analysis: Principles and Applications. New York, John Wiley \& Sons Inc., 2000. 479p.

WU, W.; FAN, Y.; WANG, Z. \& LIU, H. Assessing effects of digital elevation model resolutions on soil-landscape correlations in a hilly area. Agric. Ecosyst. Environ., 126:209-216, 2008. 